\title{
periwinkle moves
}

i want fields

of purple daisies

saffron tears

and turquoise tangerines

soft earth and crackling sky

to keep my hand moving

i want morning stories,

songs of stolen glory

i want sweetest day

i want the moon

i want to sweep up the stars,

drop them in my pocket

and keep my hand moving

monna mediarmid @2000

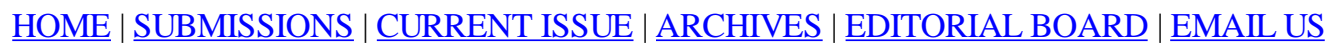

